\title{
Efficient Model Order Reduction of Structural Dynamic Systems with Local Nonlinearities under Periodic Motion
}

\author{
M. Mohammadali and H. Ahmadian \\ Center of Excellence in Experimental Solid Mechanics and Dynamics, School of Mechanical Engineering, \\ Iran University of Science and Technology, Narmak, Tehran 16844, Iran
}

Correspondence should be addressed to M. Mohammadali; mohammadali@iust.ac.ir

Received 20 October 2012; Accepted 19 November 2012; Published 9 June 2014

Academic Editor: Hamid Mehdigholi

Copyright ( 2014 M. Mohammadali and H. Ahmadian. This is an open access article distributed under the Creative Commons Attribution License, which permits unrestricted use, distribution, and reproduction in any medium, provided the original work is properly cited.

\begin{abstract}
In many nonlinear structural systems, compared with the local regions with induced nonlinear effects, the main portions of the structures are linear. An exact condensation technique based on the harmonic balance method (HBM) in conjunction with the modal expansion technique is employed to convert the motion equations of such a system to a set of nonlinear algebraic equations that are considerably small and adequately accurate to determine periodic responses. To demonstrate the capability of the suggested method, few case studies consisting of discrete systems with weak and essential nonlinearities are studied, and the results are compared to other methodologies results.
\end{abstract}

\section{Introduction}

Compared with the local regions with induced nonlinear effects, the main portions of the structural systems are linear. Typical engineering examples of these localized nonlinear sources are friction and vibroimpact in joints, local buckling, crack, nonlinear isolator, dead zone (gap), and squeeze film dampers in mechanical systems. Consequently, the dynamic response of such a system is nonlinear. A dynamic model for this structural system is useful for detailed study, prediction of response, identification of unknown parameters, or health monitoring of the overall system. Therefore, much time and effort are consumed in the development of this dynamic model that includes desired effects. Usually, the dynamic properties of the structure components are known (analysed or tested) as separate components, and they are used for development of the dynamic model for the total assembled system.

Any nonlinear response analysis involves significant computational effort, especially if the full dynamic model is used and a set of performance characteristics related to temperature, preload, deflection, and so forth characterize the nonlinear elements. Although there is significant motivation to develop a set of model order reduction (MOR) techniques to project the system model to a condensed space and considerably reduce time of nonlinear response computation, accuracy of these MOR techniques and solution algorithms are still issues. For reduction of nonlinear discrete models one can apply linear MOR techniques [1] such as Guyan reduction [2,3], improved reduced system (IRS) [4], iterated improved reduced system (IIRS) [5], system equivalent reduction expansion process (SEREP), [6] or component mode synthesis (CMS) $[4,7]$.

Other reduction methods that can be applied to reduce continuous and discrete models size are commonly used linear normal modes (LNMs) of linearized system and nonlinear normal modes (NNMs). Generation of NNMs for a small system requires considerable effort $[8,9]$, especially in the presence of internal resonance [10] and/or external excitation [11]. In the case of a large system, it is possible to use other reduction techniques and then generate NNMs of the reduced order model [12].

Reduction techniques based on linear MOR techniques, in best, will lead to an acceptable answer for weakly nonlinear system, and by growing the nonlinearity their accuracy will be lost due to their linear nature. Local equivalent linear stiffness method (LELMS) was proposed [13] to reduce the nonlinear discrete models size. In this method, an equivalent linear 
system of the nonlinear system is iteratively calculated whose mode shapes are used to reduce the system size.

There are many different algorithms for solving nonlinear systems. A semianalytical method that is generally valid over a much larger domain compared to the other methodologies is the harmonic balance method (HBM) [14]. The HBM can solve many types of nonlinear problems in the form of periodic response. The HBM is used for handling nonlinear problems consisting of forced excited, strongly nonlinear response, chaotic behaviour, and internal resonance and has a great potential for identification and health monitoring. Furthermore, this technique is applied to estimate NNMs of nonlinear systems [8]. HBM expands the periodic response of the nonlinear system in the form of truncated Fourier series, whose coefficients are estimated by solving a set of nonlinear algebraic equations. In the absence of internal resonance in many cases, the solution of one term harmonic balance has good correlation with the recorded experimental results [15]. Typically, more accurate approximation to the solution obtained by using a Fourier series with a higher number of terms and, in general, the amplitudes of the higher terms gradually decrease. Consequently, in experiment if the higher term's amplitudes are small, then inclusion of measurement noise will result in the difference between these terms in the analysis and experiment.

HBM is improved in different ways because of its capability. Incremental harmonic balance (IHB) [16] suggests a new formulation based on HBM for achieving frequency responses of nonlinear systems. With the aim of reducing the computational cost and preserving the accuracy, adaptive HBM (AHBM) [17] has proposed selection algorithms for the number of Fourier series terms. Recently, a new exact reduction technique $[18,19]$ was proposed for discrete systems that condense the problem into the nonlinear DOFs only.

The aim of this work is to study the technique suggested by KIM $[18,19]$ and extend this by expanding the nonlinear DOFs motions by natural modes of the condensed system. To demonstrate the capability of the suggested method, few case studies consisting of a discrete system with weak and essential nonlinearity are studied and the results are compared.

\section{Formulation}

The general form of a nonlinear dynamic system with $N$ degrees of freedom (DOFs) is shown below:

$$
\mathbf{M} \ddot{\mathbf{q}}+\mathbf{C} \dot{\mathbf{q}}+\mathbf{K q}+\mathbf{f}_{\mathrm{nl}}=\mathbf{f}_{E}
$$

where $\mathbf{q}$ is the field deformation vector, $\mathbf{M}, \mathbf{C}$, and $\mathbf{K}$ are mass, damping, and stiffness matrices, $\mathbf{f}_{\mathrm{nl}}$ is nonlinear force, and $\mathbf{f}_{E}$ is periodic excitation force. $\mathbf{q}, \mathbf{f}_{\mathrm{nl}}$, and $\mathbf{f}_{E}$ are function of time which time argument, $t$, is dropped for convenience. If the steady-state solution of above equation with the period of $T=2 \pi / \omega$ is requested, the deformation field can be expanded with the aid of HBM in the form of

$$
\mathbf{q}=\sum_{j=0}^{m}\left(\psi_{j} \cos (j \omega t)+\boldsymbol{\varphi}_{j} \sin (j \omega t)\right),
$$

where $\psi_{j}$ and $\varphi_{j}$ are vectors of vibration amplitude at the frequency of $j \omega$. By substituting (2) into (1) and using Galerkin method, the equation of motion is decoupled in the form below:

$$
\begin{gathered}
-j^{2} \omega^{2} \mathbf{M} \boldsymbol{\psi}_{j}+j \omega \mathbf{C} \boldsymbol{\varphi}_{j}+\mathbf{K} \boldsymbol{\psi}_{j}+\mathbf{f}_{\mathrm{nl}, c_{j}}=\mathbf{f}_{E, c_{j}}, \\
-j^{2} \omega^{2} \mathbf{M} \boldsymbol{\varphi}_{j}-j \omega \mathbf{C} \psi_{j}+\mathbf{K} \boldsymbol{\varphi}_{j}+\mathbf{f}_{\mathrm{nl}, s_{j}}=\mathbf{f}_{E, s_{j}}, \\
j=0,1,2, \ldots, m, \\
\left\{\mathbf{f}_{X, c_{j}}, \mathbf{f}_{X, s_{j}}\right\}=(1+\operatorname{sgn}(j)) \\
\times \frac{\omega}{2 \pi} \int_{0}^{2 \pi / \omega}\left\{\mathbf{f}_{X} \cos (j \omega t), \mathbf{f}_{X} \sin (j \omega t)\right\} d t
\end{gathered}
$$

where $X$ is replaced by arbitrary subscripts of force vectors. Equation (3) expresses one static equilibrium and $2 m$ dynamic equations. By using imaginary unit $\iota=\sqrt{-1}$, (3) can be put together in the form

$$
\begin{array}{r}
\left(-j^{2} \omega^{2} \mathbf{M}-\iota j \omega \mathbf{C}+\mathbf{K}\right) \boldsymbol{\chi}_{j}+\mathbf{f}_{\mathrm{nl} j}=\mathbf{f}_{E j} \\
j=0,1,2, \ldots, m,
\end{array}
$$

where

$$
\begin{gathered}
\chi_{j}=\psi_{j}+\iota \boldsymbol{\varphi}_{j}, \\
\mathbf{f}_{X j}=\mathbf{f}_{X, c_{j}}+\iota \mathbf{f}_{X, s_{j}} .
\end{gathered}
$$

In the case of localized nonlinearity, it is possible to write nonlinear force vector in the form

$$
\begin{gathered}
\mathbf{f}_{\mathrm{nl}}^{T}=\left\{f_{\mathrm{nl} l}, \ldots, f_{\mathrm{nl} p}, 0,0, \ldots, 0\right\}, \\
\mathbf{f}_{\mathrm{nl}}=\left\{\begin{array}{c}
\mathbf{f}_{\mathrm{nl}_{\alpha}} \\
\mathbf{0}
\end{array}\right\} .
\end{gathered}
$$

Furthermore, the deformation field can be divided into two vectors with subscripts $\alpha$ and $\beta$, which stand for active or master coordinates and deleted or slave coordinates, respectively. Active coordinates are experiencing nonlinear forces and deleted coordinates are only subjected to linear forces. Also, $\mathbf{f}_{E}$ vector and $\mathbf{M}, \mathbf{C}$, and $\mathbf{K}$ matrices can be divided appropriately with subscripts $\alpha$ and $\beta$. Therefore, the motion equation in (4) can be written in the form

$$
\begin{gathered}
\left(-j^{2} \omega^{2}\left[\begin{array}{ll}
\mathbf{M}_{\alpha \alpha} & \mathbf{M}_{\alpha \beta} \\
\mathbf{M}_{\beta \alpha} & \mathbf{M}_{\beta \beta}
\end{array}\right]-\iota j \omega\left[\begin{array}{ll}
\mathbf{C}_{\alpha \alpha} & \mathbf{C}_{\alpha \beta} \\
\mathbf{C}_{\beta \alpha} & \mathbf{C}_{\beta \beta}
\end{array}\right]\right. \\
\left.+\left[\begin{array}{ll}
\mathbf{K}_{\alpha \alpha} & \mathbf{K}_{\alpha \beta} \\
\mathbf{K}_{\beta \alpha} & \mathbf{K}_{\beta \beta}
\end{array}\right]\right)\left\{\begin{array}{l}
\boldsymbol{\chi}_{\alpha_{j}} \\
\boldsymbol{\chi}_{\beta_{j}}
\end{array}\right\}+\left\{\begin{array}{c}
\mathbf{f}_{\mathrm{nl}_{\alpha}} \\
\mathbf{0}
\end{array}\right\} \\
=\left\{\begin{array}{c}
\mathbf{f}_{E_{\alpha}} \\
\mathbf{f}_{E_{\beta}}
\end{array}\right\}, \quad j=0,1,2, \ldots, m .
\end{gathered}
$$




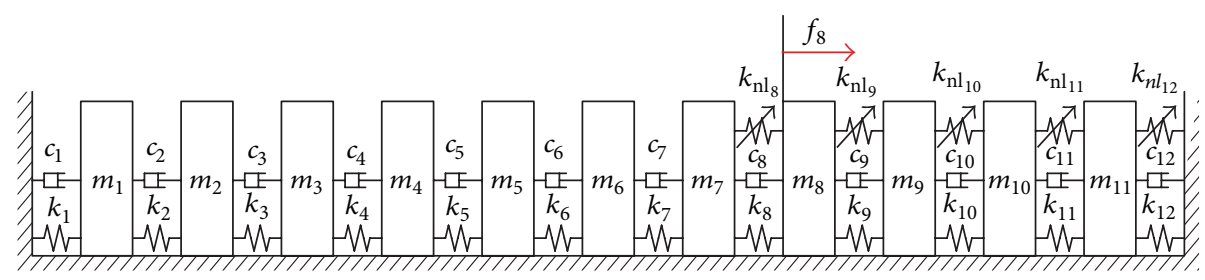

FIGURE 1: The discrete system model.

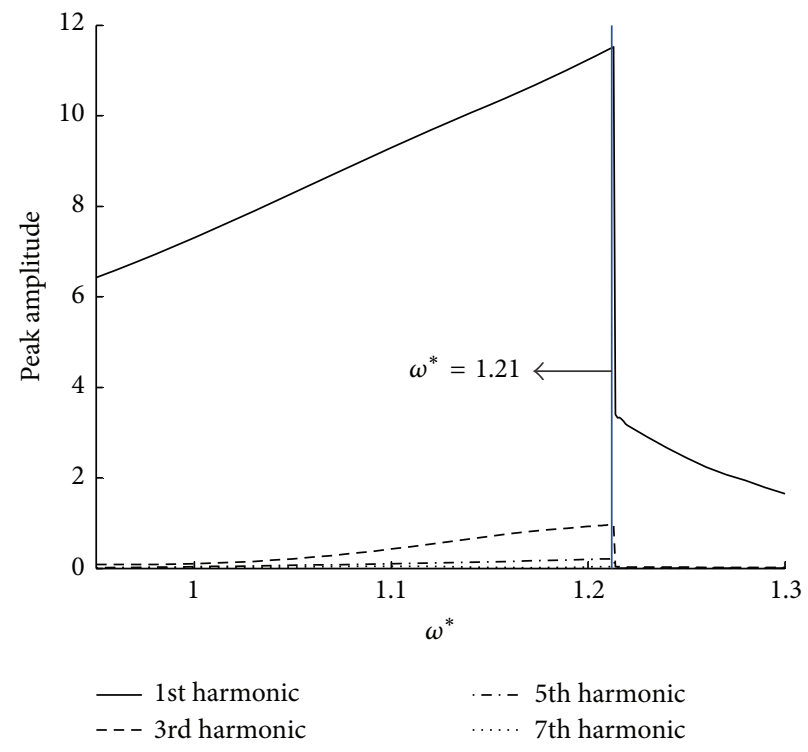

(a)

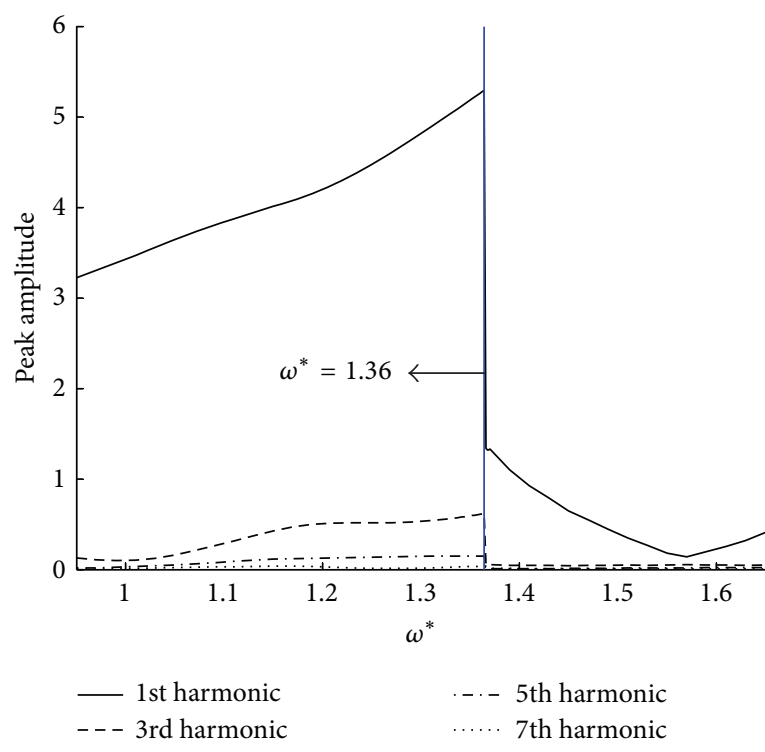

(b)

FIGURE 2: The response peak amplitudes of each harmonic at 8th DOF: (a) weakly nonlinear system; (b) essentially nonlinear system.

In above equation, one can eliminate $\chi_{\beta_{j}}$ from the first row with the aid of the second row; this will result in exact reduced form of motion equation

$$
\begin{gathered}
\left(-j^{2} \omega^{2} \mathbf{M}_{\alpha \alpha}-\imath j \omega \mathbf{C}_{\alpha \alpha}+\mathbf{K}_{\alpha \alpha}-\mathbf{K}_{M j}\right) \boldsymbol{\chi}_{\alpha_{j}}+\mathbf{f}_{\mathrm{n}_{\alpha j}} \\
=\mathbf{f}_{E_{\alpha_{j}}}-\mathbf{f}_{E T_{\beta_{j}}}, \\
\mathbf{A}_{j}=\left(-j^{2} \omega^{2} \mathbf{M}_{\alpha \beta}-\imath j \omega \mathbf{C}_{\alpha \beta}+\mathbf{K}_{\alpha \beta}\right) \\
\times\left(-j^{2} \omega^{2} \mathbf{M}_{\beta \beta}-\imath j \omega \mathbf{C}_{\beta \beta}+\mathbf{K}_{\beta \beta}\right)^{-1} \\
\mathbf{K}_{M j}=\mathbf{A}_{j}\left(-j^{2} \omega^{2} \mathbf{M}_{\beta \alpha}-\imath j \omega \mathbf{C}_{\beta \alpha}+\mathbf{K}_{\beta \alpha}\right), \\
\mathbf{f}_{E T_{\beta_{j}}}=\mathbf{A}_{j} \mathbf{f}_{E_{\beta_{j}}} .
\end{gathered}
$$

The deformation field vectors, $\boldsymbol{\chi}_{\alpha_{j}}$, can be expanded by one of its basis. Orthogonal bases which consist of arranged vectors with minimum energy required for excitation will be suitable bases to approximate the deformation field vectors and reduce the size of condense system. To find such bases,
$\mathbf{K}_{M j}$ can be considered as a constant stiffness matrix, whose dependence on frequency, $j \omega$, is neglected; then

$$
\left(-\lambda_{j k}^{2} \mathbf{M}_{\alpha \alpha}-\imath \lambda_{j k} \mathbf{C}_{\alpha \alpha}+\mathbf{K}_{\alpha \alpha}-\mathbf{K}_{M j}\right) \phi_{j k}=\mathbf{0}
$$

is the eigenvalue problem of the linearized condensed system, where $\phi_{j k}$ is the $k$ th mass normalized, maximum real right eigenvector and $\lambda_{j k}$ is its companion eigenvalue. The $\phi_{j k}$ are orthogonal basis of $\chi_{\alpha_{j}}$ and by increasing the $k$ index the required energy for excitation $\phi_{j k}$ in linearized condensed system will grow. Considering that the motion of the nonlinear condensed system is close to linearized system, the deformation field in (8) can be approximated by few vectors of these orthogonal bases. Therefore, the deformation field can be written in the form

$$
\chi_{\alpha_{j}} \cong \Phi_{j}\left(\boldsymbol{\eta}_{c_{j}}+\boldsymbol{\eta}_{s_{j}}\right)
$$

where $\boldsymbol{\eta}_{c_{j}}$ and $\boldsymbol{\eta}_{s_{j}}$ are unknown real vectors and $\boldsymbol{\Phi}_{j}$ is the modal matrix in which collaborated mode shapes, $\phi_{j k}$, are arranged in column fashion. After substituting (10) into (8) 


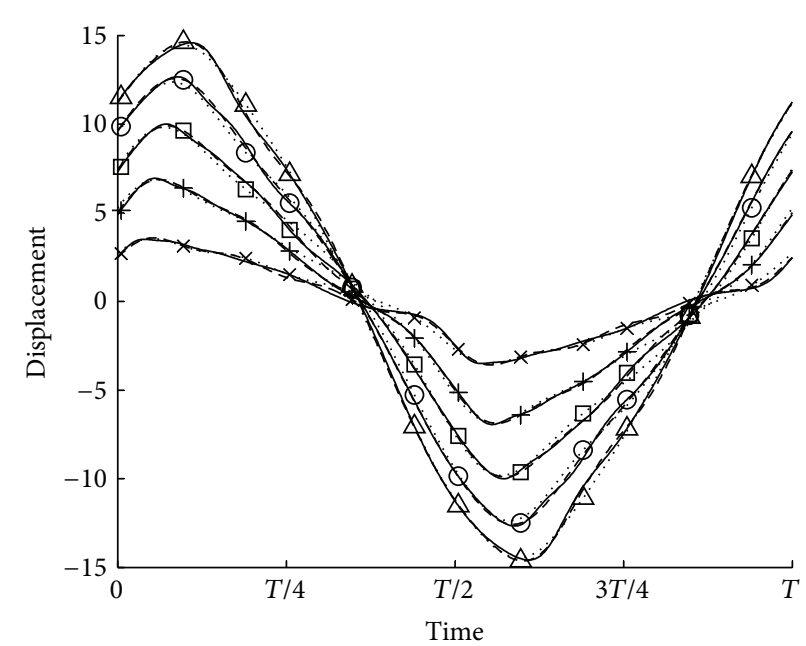

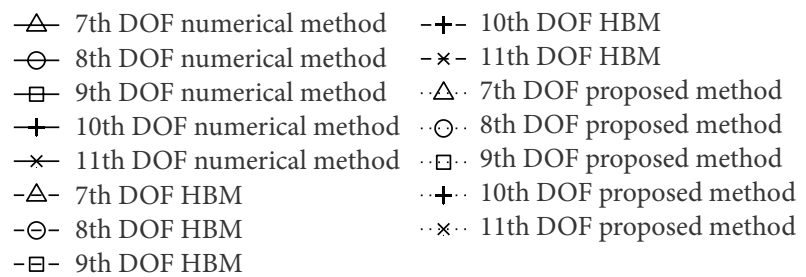

(a)
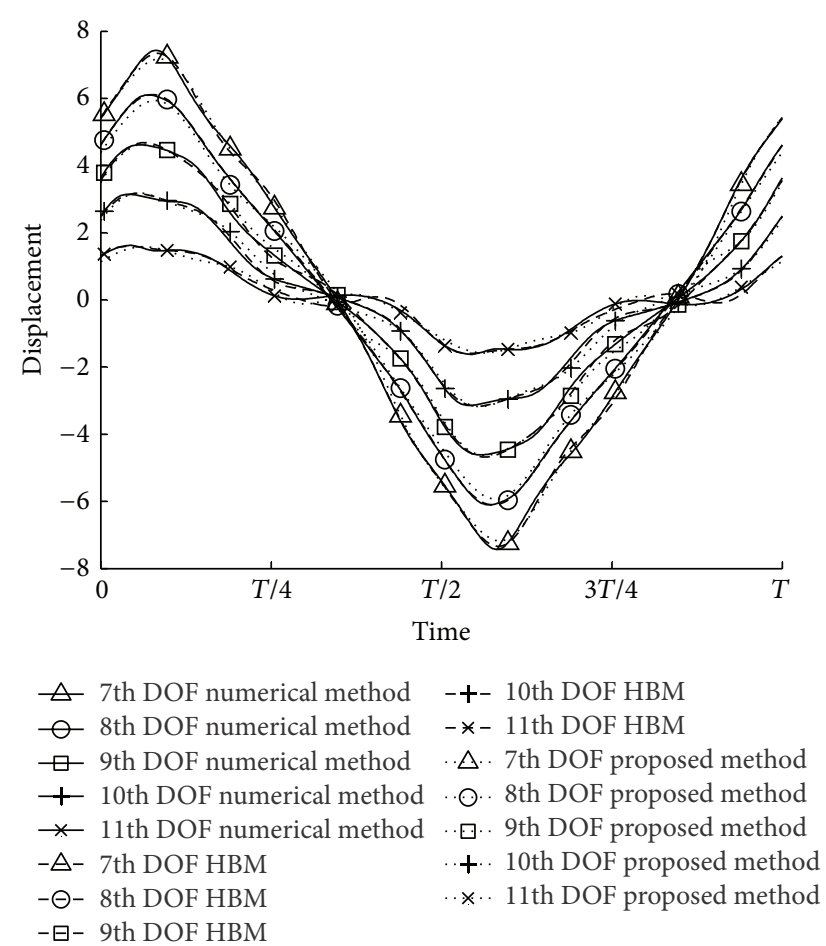

(b)

FIGURE 3: Time response of nonlinear systems at their resonance: (a) weakly nonlinear system $\omega^{*}=1.21$; (b) essentially nonlinear system $\omega^{*}=1.36$.

and using Galerkin method the motion equation will become in the form

$$
\begin{gathered}
\boldsymbol{\Theta}_{j}\left(\boldsymbol{\eta}_{c_{j}}+\imath \boldsymbol{\eta}_{s_{j}}\right)+\left\langle\mathbf{f}_{\mathrm{nl}_{\alpha j}}, \boldsymbol{\Phi}_{j}\right\rangle=\left\langle\mathbf{f}_{E_{\alpha j}}-\mathbf{f}_{E T_{\beta_{j}}}, \boldsymbol{\Phi}_{j}\right\rangle, \\
\boldsymbol{\Theta}_{j}=\left\langle\left(-j^{2} \omega^{2} \mathbf{M}_{\alpha \alpha}-\imath j \omega \mathbf{C}_{\alpha \alpha}+\mathbf{K}_{\alpha \alpha}-\mathbf{K}_{M_{j}}\right) \boldsymbol{\Phi}_{j}, \boldsymbol{\Phi}_{j}\right\rangle, \\
\langle\mathbf{a}, \mathbf{b}\rangle=\mathbf{b a},
\end{gathered}
$$

where $*$ is transpose operator. The real and imaginary part of above equation will result in two different nonlinear equations. Therefore,

$$
\begin{gathered}
{\left[\begin{array}{cc}
\operatorname{Re}\left(\boldsymbol{\Theta}_{j}\right) & -\operatorname{Im}\left(\boldsymbol{\Theta}_{j}\right) \\
\operatorname{Im}\left(\boldsymbol{\Theta}_{j}\right) & \operatorname{Re}\left(\boldsymbol{\Theta}_{j}\right)
\end{array}\right]\left\{\begin{array}{l}
\boldsymbol{\eta}_{c_{j}} \\
\boldsymbol{\eta}_{s_{j}}
\end{array}\right\}+\left\{\begin{array}{l}
\operatorname{Re}\left(\left\langle\mathbf{f}_{\mathrm{nl}_{\alpha_{j}}}, \boldsymbol{\Phi}_{j}\right\rangle\right) \\
\operatorname{Im}\left(\left\langle\mathbf{f}_{\mathrm{nl}_{\alpha_{j}}}, \boldsymbol{\Phi}_{j}\right\rangle\right)
\end{array}\right\}} \\
\quad=\left\{\begin{array}{l}
\operatorname{Re}\left(\left\langle\mathbf{f}_{E_{\alpha_{j}}}-\mathbf{f}_{E T_{\beta_{j}}}, \boldsymbol{\Phi}_{j}\right\rangle\right) \\
\operatorname{Im}\left(\left\langle\mathbf{f}_{E_{\alpha_{j}}}-\mathbf{f}_{E T_{\beta_{j}}}, \boldsymbol{\Phi}_{j}\right\rangle\right)
\end{array}\right\} .
\end{gathered}
$$

By solving (12) with an arbitrary method the final solution of motion will be achieved.

\section{Numerical Results}

In what follows, to prove the power of the proposed reduction technique discrete vibrating systems are studied with weak and essential nonlinearities of the cubic form. These systems have 11 DOFs in which 5 of its DOFs are connected together with nonlinear stiffness (Figure 1).

The steady-state responses of the system under periodic excitation are determined by the numerical method, HBM, and proposed method. The resultant nonlinear algebraic equations of HBM and proposed method are solved by Newton-Raphson algorithm. The computational complexity of this method at each step can be approximated by the square of the number of equations.

The motion of these systems is governed by

$$
\begin{gathered}
m \ddot{x}_{i}+c\left(2 \dot{x}_{i}-\dot{x}_{i+1}-\dot{x}_{i-1}\right)+k\left(2 x_{i}-x_{i+1}-x_{i-1}\right) \\
+k_{\mathrm{nl} i}\left(x_{i}-x_{i-1}\right)^{3}+k_{\mathrm{nl} i+1}\left(x_{i}-x_{i+1}\right)^{3}=f_{i}
\end{gathered}
$$

where $i=1,2, \ldots, 11, m=1, c=1 / 10, k=1$, and

$$
f_{i}= \begin{cases}0 & i \neq 8 \\ \cos (\omega t) & i=8 .\end{cases}
$$

For the case of weak and essential nonlinearities $k_{\mathrm{nl} i}$ is taken as

$$
\left\{\begin{array} { l l } 
{ 0 } & { i < 7 } \\
{ \frac { 1 } { 5 } } & { i \geq 7 , }
\end{array} \quad \left\{\begin{array}{ll}
0 & i<7 \\
2 & i \geq 7
\end{array}\right.\right.
$$

respectively. $\omega_{1}=0.26 \mathrm{~Hz}$, real part of the first resonance frequency of linearized system, is used to normalize the excitation frequency $\omega^{*}=\omega / \omega_{1}$. Peak amplitudes of each 
harmonic at 8th DOF are shown in Figure 2 for different frequencies.

The steady-state responses of the systems at their resonances are approximated by first 7 harmonies.

The nonlinear systems with $11 \mathrm{DOF}$ are reduced to 5 master DOFs and solved using HBM, which causes around $80 \%$ decrease in computational cost. By applying the proposed method to the condensed system and expanding the response using its first three mode shapes, another $64 \%$ computational cost reduction is achieved due to reduction of systems size from 5 DOFs to 3 DOFs. The results are shown in Figure 3.

In both cases, there are negligible differences between numerical method and the HBM due to harmonic truncation order; therefore, this error is present in the proposed method results.

Compared to the results of the HBM and numerical method, phase of the DOFs vibration computed by the proposed method is accurate in both nonlinear systems. However, the difference between the calculated amplitudes with the proposed and other methods is increased with growing the nonlinearity, but the captured response is still acceptable. These differences are due to reduction of exact condensed system size, from 5 DOFs to 3 DOFs, which leads to a stiffer system.

\section{Conclusion}

In this paper, a new condensation technique is suggested for discrete systems by the combination of exact condensation techniques based on HBM and modal expansion technique to reduce the computational cost and preserve the accuracy. The proposed method is used to reduce the size of two nonlinear systems with weak and essential nonlinearity. At last, the predicted periodic responses are compared with other methodologies results. The correlation between the results of the proposed method and other techniques is appropriate. Using the suggested technique, the reduced form of the system is independent of system nonlinearities; therefore, this technique has a great potential for the identification of system nonlinearities. The main drawback of the suggested technique is its frequency dependency.

\section{Conflict of Interests}

The authors declare that there is no conflict of interests regarding the publication of this paper.

\section{References}

[1] Z. Qu, Model Order Reduction Techniques, 2004.

[2] R. J. Guyan, "Reduction of stiffness and mass matrices," AIAA Journal, vol. 3, no. 2, pp. 380-380, 1965.

[3] R. W. Stephenson and K. W. Rouch, "Modeling rotating shafts using axisymmetric solid finite elements with matrix reduction," Journal of Vibration and Acoustics, Transactions of the ASME, vol. 115, no. 4, pp. 484-489, 1993.

[4] M. I. Friswell, J. E. T. Penny, and S. D. Garvey, "Using linear model reduction to investigate the dynamics of structures with local non-linearities," Mechanical Systems and Signal Processing, vol. 9, no. 3, pp. 317-328, 1995.

[5] M. I. Friswell, S. D. Garvey, and J. E. T. Penny, "Model reduction using dynamic and iterated IRS techniques," Journal of Sound and Vibration, vol. 186, no. 2, pp. 311-323, 1995.

[6] J. O'Callahan, P. Avitabile, and R. Riemer, "System equivalent reduction expansion process (SEREP)," Tech. Rep., IMAC, 1989.

[7] M. C. C. Bampton and R. R. Craig, "Coupling of substructures for dynamic analyses," AIAA Journal, vol. 6, no. 7, pp. 1313-1319, 1968.

[8] G. Kerschen, M. Peeters, J. C. Golinval, and A. F. Vakakis, "Nonlinear normal modes, Part I: a useful framework for the structural dynamicist," Mechanical Systems and Signal Processing, vol. 23, no. 1, pp. 170-194, 2009.

[9] M. Peeters, R. Viguié, G. Sérandour, G. Kerschen, and J.-C. Golinval, "Nonlinear normal modes, Part II: toward a practical computation using numerical continuation techniques," Mechanical Systems and Signal Processing, vol. 23, no. 1, pp. 195216, 2009.

[10] D. Jiang, C. Pierre, and S. W. Shaw, "The construction of nonlinear normal modes for systems with internal resonance," International Journal of Non-Linear Mechanics, vol. 40, no. 5, pp. 729-746, 2005.

[11] D. Jiang, C. Pierre, and S. W. Shaw, "Nonlinear normal modes for vibratory systems under harmonic excitation," Journal of Sound and Vibration, vol. 288, no. 4-5, pp. 791-812, 2005.

[12] M. Legrand, D. Jiang, C. Pierre, and S. Shaw, "Nonlinear normal modes of a rotating shaft based on the invariant manifold method," The International Journal of Rotating Machinery, vol. 10, no. 4, pp. 319-335, 2004.

[13] E. A. Butcher, "Clearance effects on bilinear normal mode frequencies," Journal of Sound and Vibration, vol. 224, no. 2, pp. 305-328, 1999.

[14] M. Peeters, G. Kerschen, and J. C. Golinval, "Dynamic testing of nonlinear vibrating structures using nonlinear normal modes," Journal of Sound and Vibration, vol. 330, no. 3, pp. 486-509, 2011.

[15] T. A. Doughty, P. Davies, and A. K. Bajaj, "A comparison of three techniques using steady state data to identify non-linear modal behavior of an externally excited cantilever beam," Journal of Sound and Vibration, vol. 249, no. 4, pp. 785-813, 2002.

[16] A. Grolet and F. Thouverez, "On a new harmonic selection technique for harmonic balance method," Mechanical Systems and Signal Processing, vol. 30, pp. 43-60, 2012.

[17] Y. B. Kim and S. T. Noah, "Response and bifurcation analysis of a MDOF rotor system with a strong nonlinearity," Nonlinear Dynamics, vol. 2, no. 3, pp. 215-234, 1991.

[18] Y. B. Kim and S. T. Noah, "Stability and bifurcation analysis of oscillators with piecewise-linear characteristics. A general approach," Journal of Applied Mechanics, Transactions ASME, vol. 58, no. 2, pp. 545-553, 1991.

[19] S. L. Lau and Y. K. Cheung, "Amplitude incremental variational principle for nonlinear vibration of elastic systems," Journal of Applied Mechanics, vol. 48, no. 4, pp. 959-964, 1981. 

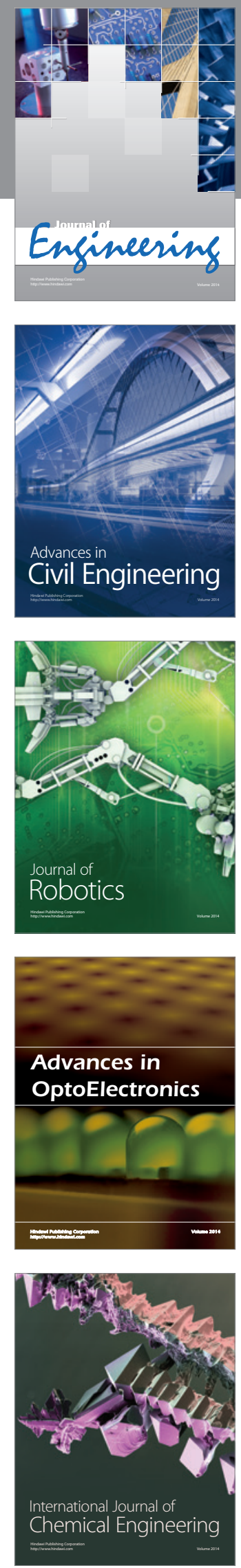

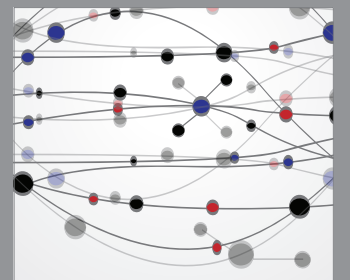

The Scientific World Journal
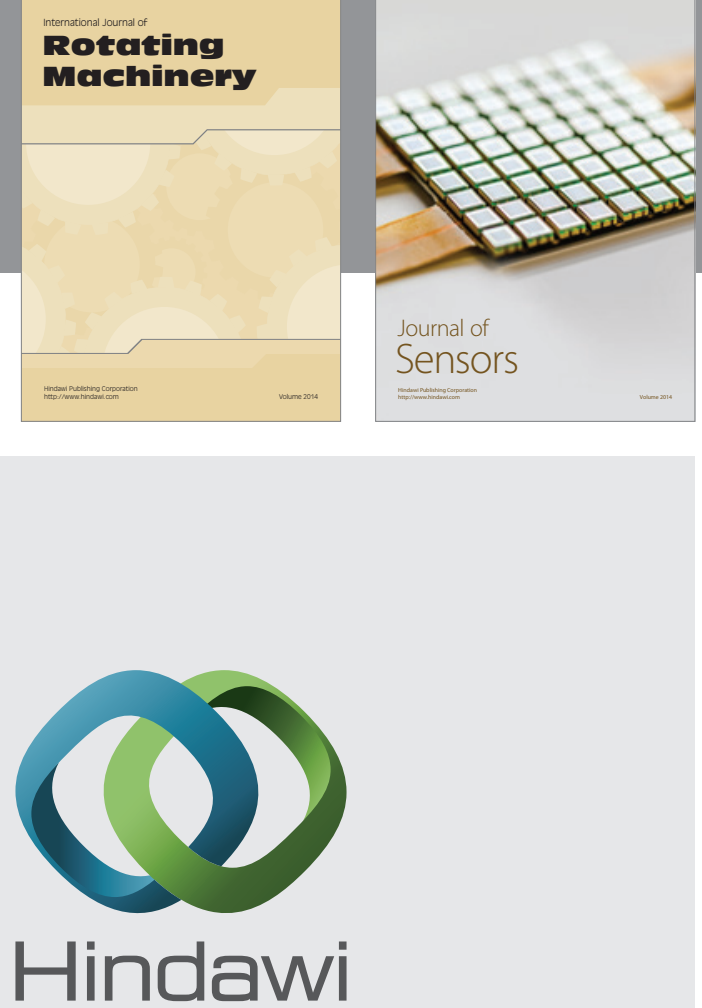

Submit your manuscripts at http://www.hindawi.com
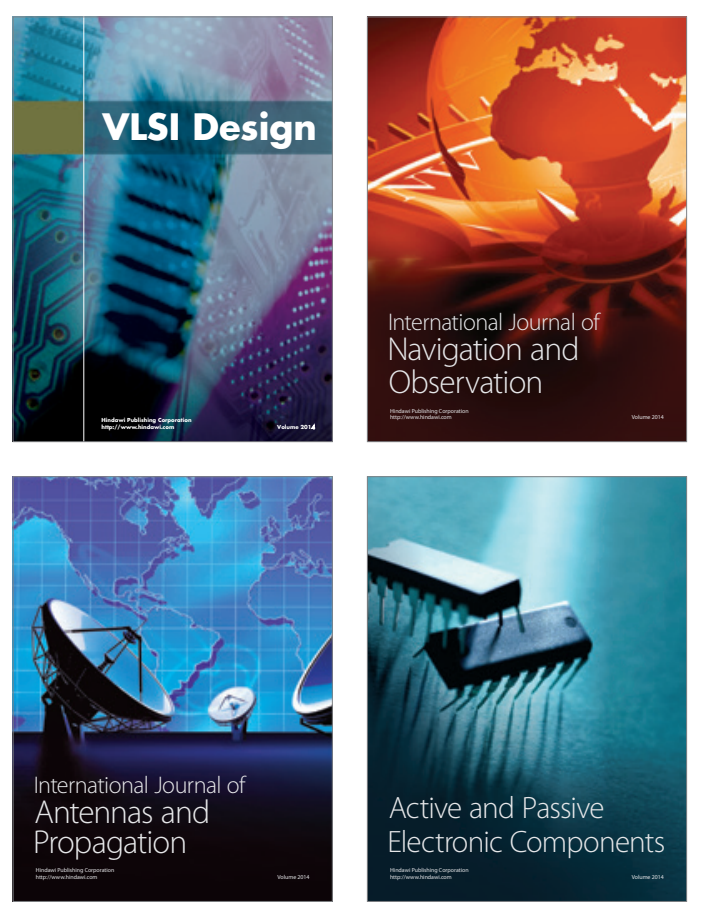
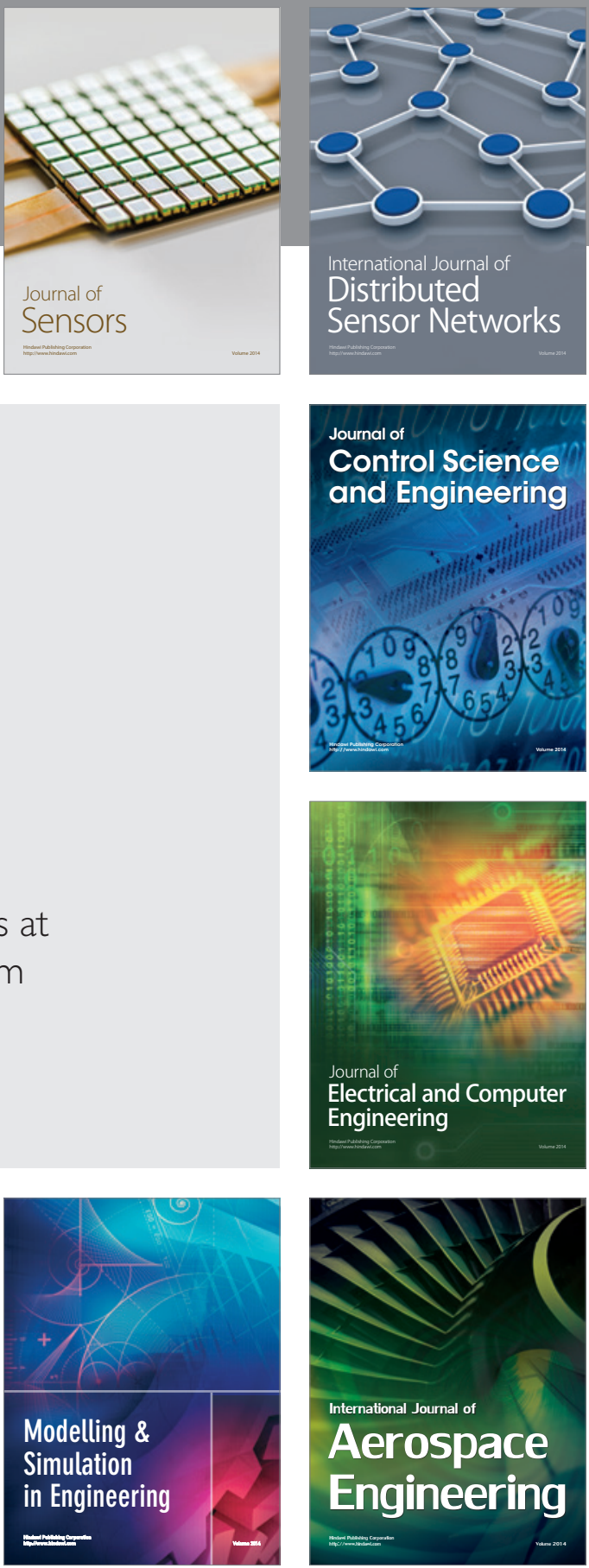

Journal of

Control Science

and Engineering
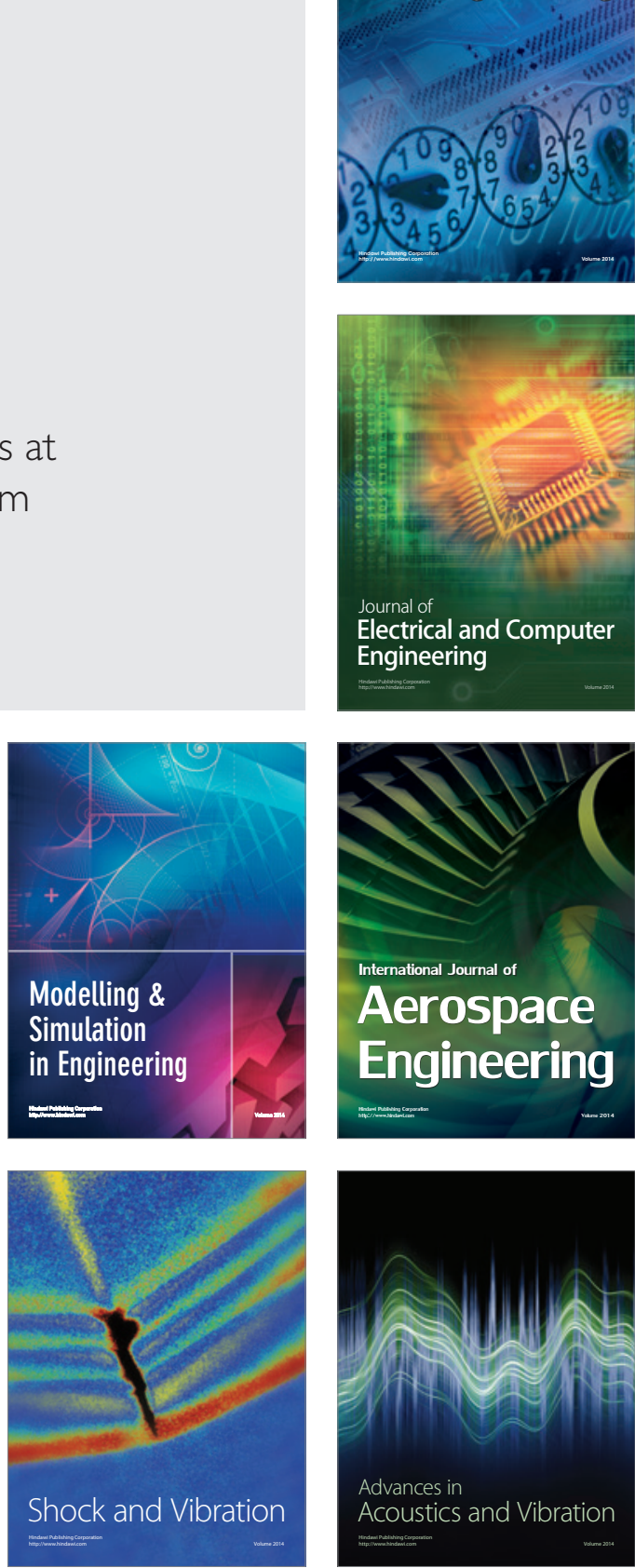